\title{
Sistemas de informacion hospitalarios: el C.M.B.D.
}

\author{
José Antonio Salvador Oliván \\ Departamento de Ciencias de la Documentación \\ e Historia de la Ciencia \\ Universidad de Zaragoza
}

\subsection{Resumen}

La sanidad pública en general, y los hospitales en particular, se encuentra ante el tremendo reto de tener que proporcionar más y mejor asistencia en un entorno de costes crecientes y presupuestos restrictivos. Indudablemente, un sistema de información que permita la toma de decisiones para optimizar la utilización de unos recursos siempre escasos y mejorar la asistencia al paciente, será la herramienta más eficaz para enfrentarse con éxito a ese reto. El eje central de este sistema de información lo constituye el Conjunto Mínimo Básico de Datos al alta hospitalaria, que se puede comportar a la vez como sistema de información para la gestión y como sistema de recuperación de información.(Autor)

Palabras clave: Sistema de información hospitalario. Conjunto Mínimo Básico de Datos.

\subsection{Abstract}

The public health, and the hospitals in particular, face up to the challenge to provide more and better medical care in rising costs and restrictive budgets environment. It is necessary an information system based on reliable and available information which let make decissions in order to optimize the poor resources, to obtain greater efficiency of health care and assure quality of care. This paper describes the core of this information system, the Hospital Discharge Minimum Data Set, which becomes the standard of hospital information systems by their aplications as management information system and information retrieval system.(Author)

Keywords: Hospital information system. Minimum Data Set. 


\section{Introducción}

El funcionamiento de un hospital, al igual que en cualquier empresa, depende de lo bien que se utilicen sus recursos. La maquinaria, material diverso, el dinero, las personas y la información son todos recursos; pero la información es, quizá, el recurso más valioso que cualquier empresa tiene, ya que sin él todos los demás recursos quedan aislados e inmanejables.

La información como recurso, es abundante en un hospital. Un diagnóstico rápido y el tratamiento eficaz de un paciente dependen de la transmisión exacta de órdenes y resultados entre varios servicios hospitalarios. Obviamente, un hospital es el marco ideal para la tecnología de comunicaciones informatizada que se utiliza tan ampliamente en las empresas de gestión. No existe un campo con una necesidad tan grande de proceso automático de datos como el de la asistencia sanitaria. La cantidad de datos que se genera aquí es inmensa, y las mejoras en la eficiencia que se pueden realizar con un sistema de proceso de datos funcionando adecuadamente se pueden medir en miles de horas/hombre y en gran cantidad de dinero ahorrado. De hecho, la instalación de tecnología informática en los hospitales ha constituido la herramienta más importante en la reducción de costes. Igualmente importante, el manejo de información médica automatizada mejora (o mejorará) significativamente la asistencia al paciente al reducir errores, acelerando el flujo de órdenes y resultados, y haciendo disponible una información más completa para la toma de decisiones.

Para Korpman (1991), la atención sanitaria es una industria de la información, ya que muchos proveedores de la salud gastan la mayor parte de su tiempo en crear o procesar información, aunque las mejoras en el uso óptimo y presentación de la información se han retrasado bastante. Esto parece extraño, considerando la importancia de la información en la sanidad, y que excepto para algunos casos aislados de informatización (laboratorios, farmacia, etc.) se haya hecho tan poco durante las últimas décadas para mejorar el proceso de información en el campo de la salud.

\section{El entorno de los Sistemas de Información hospitalarios}

La demanda de una información precisa y oportuna está creciendo cada vez más en los hospitales, debido quizá al entorno tan complejo en el que funciona un hospital. Son muchas las áreas donde se pueden implantar los sistemas de información. Mass (1984) identifica cuatro grandes áreas en función de las necesidades de información: 1) información externa sobre el entorno, que incluye datos de planificación, información de precios, información para tomar decisiones políticas, datos de mercado, etc.; 2) información financiera interna, que incluye contabilidad y facturación; 3) información administrativa y de gestión, al objeto 
de medir la productividad y el rendimiento; y 4) información clínica, que incluye ingresos, su programación, peticiones de pruebas, resultados de tests, etc.

Las ventajas de la tecnología de la información/informática en un hospital son obvias: a) acumular y organizar la información para que sea accesible inmediatamente; b) mejorar la productividad al reducir el flujo de papel y la redundancia de la información; c) ayudar en la planificación de recursos y mejora del funcionamiento hospitalario; d) permitir hacer revisiones de calidad de la asistencia; y para los profesionales de la medicina (Association Of American Medical Colleges, 1986) e) gestionar la información para tratar a los pacientes más eficientemente, dejar más tiempo libre a los profesionales de la medicina para la investigación, atención al paciente, etc., mejorar el proceso educativo a través de la incorporación de la tecnología de la información y de sistemas expertos, y ampliar y racionalizar la experiencia clínica en la educación médica.

Aún cuando la situación actual del grado de informatización de los hospitales en España ha mejorado ostensiblemente sobre todo a partir de 1992 en el que se establece la implantación del Conjunto Mínimo Básico de Datos, y con los sistemas de contabilidad analítica, no deja de ser paradójico que una área de tanta importancia social y tan necesitada de sistemas eficientes de información esté todavía en la actualidad en nuestro país en unos niveles de informatización bastante más bajos que los correspondientes a otras áreas de actividad, que tienen una repercusión mucho menos directa sobre algo tan importante como es la salud, el bienestar y la calidad de vida de los ciudadanos. Es posible que esta situación sea debida, entre otras causas, a la extraordinaria complejidad y dinamismo de la actividad hospitalaria, lo que ha hecho que los métodos tradicionales de desarrollo informático no hayan dado resultados satisfactorios al aplicarlos a la problemática del hospital. Esto llevó al intento de solucionar los problemas de cada uno de los departamentos por medio de paquetes cerrados y de las propias personas del servicio (no informáticos), que junto a la aparición de los ordenadores personales, han permitido solucionar una pequeña parte de sus necesidades de información, pero a cambio ha producido la proliferación de sistemas informáticos desperdigados que conducen inevitablemente a la desintegración y a la ineficacia de las soluciones, por la falta de aprovechamiento a nivel global de datos y resultados de aplicaciones particulares.

\section{Tipos de información hospitalaria}

En un hospital se genera una gran cantidad de información, que podemos clasificarla en tres tipos:

- Administrativa: relacionada con la gestión de recursos del hospital, tanto humanos como materiales o monetarios. 
- Médico-administrativa: relacionada con la gestión de los pacientes, y consiste, por tanto, en los datos que genera un paciente como usuario de unos servicios hoteleros, hosteleros y de cuidados asistenciales en un hospital.

- Clínica: aquella que hace referencia al estado de salud o de enfermedad del paciente, y se refleja en la historia clínica, como puede ser los antecedentes personales y familiares, patología actual expresada en diagnósticos, tratamientos efectuados, resultados de pruebas diagnósticas, etc.

La información de tipo administrativo o asistencial, proporciona datos válidos tanto para la gestión y organización de un servicio clínico como para la gestión y control de un servicio administrativo, siendo de interés para los Gerentes y Administradores de los hospitales, mientras que la de tipo clínico debe de ser útil para el médico y personal sanitario. En la actualidad los sistemas de información de gestión están mucho más desarrollados por varias razones: se han potenciado desde los niveles superiores, los datos de gestión son más fáciles de estandarizar que los datos de los pacientes, los datos administrativos son más fáciles de adquirir y de documentar que los datos médicos (excepto los producidos automáticamente por los subsistemas de laboratorio, farmacia, etc.), los efectos de proceso de datos parecen ser más fáciles de medir en el área de gestión hospitalaria que el área de atención al paciente. Mientras que los sistemas de información clínicos (informatización de la historia clínica) han planteado siempre numerosos problemas, como pueden ser la falta de comunicación y entendimiento entre el médico y los informáticos, dificultad de trasladar la historia manual a formato por ordenador, falta de uniformidad en las historias, etc.; aunque se han desarrollado muchos proyectos no ha conseguido implantarse uno de forma clara para lo que constituye la historia clínica completa. Pero si a la información administrativa le unimos la información clínica, se obtiene la información suficiente para cumplir con la finalidad básica de la información hospitalaria: asesorar al clínico en su práctica diaria y realización de estudios científicos, facilitar la realización de estudios dentro del marco de la epidemiología, y apoyar la gestión del centro y de los propios servicios (Gejo, 1990).

La información clínico-asistencial debe de estar a disposición de todo aquel que la necesite para realizar su trabajo asistencial, científico, de gestión o planificación, etc., siempre y cuando queden garantizados ciertos aspectos, como salvaguardar el derecho del paciente a su intimidad, velar por la confidencialidad de los datos, y hacer un buen uso de la información, sin perjudicar al centro o a los propios pacientes. 


\section{Historia y evolución de los Sistemas de Información Hospitalarios}

La historia de los SI Hospitalarios es en realidad la historia de los SI en el campo de la salud, ya que fue el entorno hospitalario donde primero se implantaron (Kaplan, 1988):

- Durante la década de los 50 y de los 60, los sistemas de información se introdujeron casi exclusivamente por necesidades financieras y de gestión económica de los centros. Estos sistemas se centraban en recoger datos demográficos del paciente y mezclarlos con datos de costes para producir facturas. Así pues, se desarrollaron sistemas de facturación y de contabilidad. Este período viene caracterizado por los grandes sistemas informáticos (mainframes), que a su vez eran muy costosos. La incapacidad de muchos hospitales para soportar estos costos tan altos, llevaron a la necesidad y al éxito de los sistemas compartidos. Un sistema de información hospitalario típico de los 60 tenía poco de sistema clínico, ya que el énfasis estaba puesto en la contabilidad, nóminas y recursos humanos. Los únicos sistemas clínicos que se desarrollaron fueron sistemas de registros de pacientes que recogían sobre todo datos con los que estudiar diagnósticos y tomar decisiones para mejorar la precisión diagnóstica, para tomar decisiones clínicas más fiables y para aumentar la comprensión de la estructura del conocimiento médico de tal manera que se pudieran tomar mejores decisiones y mejores métodos de diagnóstico.

- En la década de los 70 comenzaron a dejarse sentir con más fuerza las necesidades clínicas, pero principalmente como un producto de las necesidades financieras. Estas cuestiones financieras se centraban en maximizar los ingresos y capturar los costes, y los sistemas de atención al paciente eran capaces de servir como vehículo para documentar las órdenes y peticiones, e indirectamente, sus costes. Sin embargo, con este objetivo financiero continuo, se hizo poco esfuerzo para tratar las necesidades de los médicos y enfermeras implicados en la atención directa al paciente. El crecimiento de los sistemas departamentales coincidió con la disponibilidad de los miniordenadores, de menor coste que los mainframes. Los hospitales estaban ya experimentando una necesidad creciente de datos de servicios específicos y del hospital en conjunto.

- La década de los 80 impulsó un cambio dramático de maximizar los ingresos a maximizar el reembolso. Se comenzó a presionar para mejorar la coordinación entre diferentes servicios y para reducir la estancia. Cada vez cobraba mayor importancia la contención de costes, los sistemas de pago prospectivo, los Grupos Relacionados por el Diagnóstico, la revisión de 
utilización, etc. Los servicios clínicos comenzaron a recibir mayor presión para mejorar la productividad. Pero un hecho clave en el crecimiento de los sistemas departamentales fue el desarrollo de los microordenadores y de los lenguajes de programación de $4^{\mathrm{a}}$ generación, que trajeron un mayor acceso a los datos a un menor coste. Un sistema de información hospitalario típico de los 80 estaba formado por un sistema de contabilidad y un sistema de atención al paciente.

- En los años que llevamos en la década de los 90, los sistemas se mueven en la dirección de centrarse sobre el paciente y estar orientados clínicamente; estos sistemas no ven al paciente como una colección de números o episodios, sino como un flujo continuo de datos. Se podrá conseguir más información desde el punto mismo de asistencia, y los sistemas mejorarán la comunicación proveedor-paciente.

Los sistemas clínicos de atención al paciente deben de tratar sobre los procesos clínicos y servir de apoyo a la toma de decisiones de los médicos. Dowling (1989) declaró que "el proceso de producción principal de un hospital es la asistencia al paciente, no el proceso financiero". De aquí, que el núcleo de la arquitectura de los sistemas de información deba de ser los procesos clínicos: valoración, planificación del alta, planificación del tratamiento, entrada de peticiones y órdenes, informes de resultados, acceso a datos clínicos del paciente, historias clínicas automatizadas y acceso a literatura médica. La conexión en el hospital de diferentes puestos de trabajo a través de redes informáticas es el presente/futuro; esta conectividad permitirá transferir datos o compartirlos entre diferentes aplicaciones; el elemento principal de la estructura de estas redes será la fibra óptica, de gran velocidad de transmisión. Existirán nodos para acceder a bases de datos externas y para comunicarse con otros hospitales u otras instalaciones; se podrán transmitir imágenes entre diferentes ciudades y países.

\section{Objetivos de los Sistemas de Información Hospitalarios}

De acuerdo con Collen (1988), los objetivos básicos de un sistema de información hospitalario son los siguientes:

- Establecer una base de datos capaz de proporcionar un registro médico integrado de datos asistenciales para todos los pacientes, y que sea accesible para todos los profesionales médicos y de la salud debidamente autorizados.

- Posibilidad de comunicar los datos del paciente a / desde todos los servicios administrativos y clínicos del hospital.

- Soportar todas las funciones del proveedor de asistencia sanitaria, incluyendo la entrada de órdenes, informes de resultados, historia del paciente, 
informes de procedimientos, etc., y comunicar datos individuales del paciente a los profesionales sanitarios.

- Proporcionar apoyo en la toma de decisiones clínica y administrativa.

- Establecer y mantener ficheros para las funciones administrativas y de gestión hospitalaria, incluyendo aplicaciones de personal, recursos, programación, registro, etc.

- Ayuda en la evaluación de la calidad, acreditación y requisitos reguladores.

- Apoyo a la investigación y educación.

Realmente, los sistemas de información hospitalarios pueden servir en todas las situaciones en las que se necesiten tomar decisiones; la literatura está llena de ejemplos, citando como ejemplos más representativos de su utilidad para los profesionales médicos los que hacen referencia a la investigación epidemiológica clínica (Zwetsloot-Schonk, 1989), en el desarrollo de recursos humanos (Who, 1990), investigación general (Gerneth, 1991), evaluación de la calidad (Selbmann, 1990), planificación (Regidor, 1991), etc.

\section{El Conjunto Mínimo Básico de Datos al Alta Hospitalaria (CMBD)}

\subsection{Antecedentes del CMBD}

La definición del Minimum Basic Data Set europeo para hospitales de agudos se realizó después de identificar los datos que los hospitales utilizaban sistemáticamente en sus sistemas de información. El Grupo de Estudio de la OMS, reunido en Ginebra en 1969, recomendó un conjunto básico de datos, con fines estadísticos, que debían ser preparados a partir de cada historia del paciente hospitalizado.

- Estados Unidos: en 1974, el National Committe on Vital and Health Statistics de EE.UU., aceptó y estableció un Conjunto Mínimo de datos al Alta Hospitalaria (Uniform Hospital Discharge Data Set) formado por 14 ítems, relativos tanto a datos de filiación y administrativos como a datos clínicos.

- Europa: en Septiembre de 1981, en Bruselas, la Workshop on Hospital Statistics for Population Based Health Care and Epidemiology, bajo el patrocinio de la Comisión de las Comunidades Europeas, la Oficina Europea de la O.M.S., el Comité Hospitalario de la Comunidad Económica Europea y la Asociación Internacional de Informática Médica, entre otras recomendaciones, y tomando como referencia los datos de Estados Unidos, planteó la aceptación de un conjunto mínimo básico de datos de 13 ítems como base para la gestión, planificación y evaluación de la asistencia y los servicios de salud y para la investigación epidemiológica y clínica. 


\begin{tabular}{|c|l|l|l|}
\hline & EEUU & CONSEJO DE EUROPA & CONSEJO INTERTERRITORIAL \\
\hline 1 & Identificación del hospital & Identificación del hospital & Identificación del hospital \\
\hline 2 & Identificación del paciente & Identificación del paciente & Identificación del paciente \\
\hline 3 & Sexo & Sexo & Sexo \\
\hline 4 & Fecha de nacimiento & Edad & Fecha de nacimiento \\
\hline 5 & Raza y etnia & Estado civil & Residencia \\
\hline 6 & Lugar de residencia & Lugar de residencia & Financiación \\
\hline 7 & Fecha de admisión & Mes y año de admisión & Fecha de ingreso \\
\hline 8 & Fecha de alta & Duración de la estancia & Circunstancias de ingreso \\
\hline 9 & Circunstancias al alta & Circunstancias al alta & Diagnóstico principal y otros \\
\hline 10 & Diagnósticos & Diagnóstico principal & Procedimientos quirúrgicos y otros. \\
\hline 11 & Procedimientos y fecha & Otros diagnósticos & Procedimientos diagnósticos \\
\hline 12 & Identificación del médico & Procedimientos quirúrgicos & Fecha del alta \\
\hline 13 & Identificación del cirujano & Otros procedimientos & Circunstancias al alta \\
\hline 14 & Financiación & & $\begin{array}{l}\text { Identificación del médico } \\
\text { responsable del alta }\end{array}$ \\
\hline
\end{tabular}

Tabla.1. Conjunto mínimo básico de datos de Estados Unidos, Europa y España

- España: en 1984 el Ministerio de Sanidad y Consumo regula la obligatoriedad del Informe de Alta, en el que se indica qué datos mínimos del episodio asistencial deben de incluirse en él (Orden de 6 de Septiembre de 1994).

En el año 1987, el Consejo Interterritorial en el Pleno celebrado el día 14 de Diciembre, aprueba establecer un Conjunto Mínimo Básico de Datos al Alta Hospitalaria, compuesto de 14 ítems.

En la tabla 1 puede observarse que en España se recoge el ítem circunstancias del ingreso, que no aparece en Europa ni en EEUU, mientras que el estado civil que contiene los datos europeos y la raza y etnia de los datos de EEUU no aparecen en el conjunto de datos españoles. Aunque los datos fundamentales de tipo administrativo y clínicos son los mismos en los tres grupos.

Entre los años 1987 y 1991, aparecen diversas experiencias piloto de implantación del CMBD en los hospitales de la red del INSALUD, encuadradas en el proyecto CODIGO, elaborado dentro del Plan de Calidad Total del Instituto Nacional de la Salud.

La primera Comunidad Autónoma que pone en marcha el CMBD es Cataluña, mediante un decreto del departamento de Sanidad y Seguridad Social de la Generalitat (Orden de Noviembre de 1986, DOGC 3-12-1986), modificado 
en 1990 (DOGC 12-12-1990), mediante el que se regula el Informe Clínico del alta hospitalaria y el Conjunto Mínimo Básico de Datos al Alta Hospitalaria para el ámbito de dicha Comunidad.

Posteriormente, el 24 de Enero de 1992, la Secretaría General del Sistema Nacional de Salud del Ministerio de Sanidad y Consumo resuelve que todos los centros hospitalarios propios y administrados por el INSALUD-GD quedan obligados al registro y codificación del CMBD al Alta Hospitalaria de todos los pacientes ingresados, y que su implantación deberá de producirse antes del 31 de diciembre de 1992. Para ello, se define la hoja Clínico-Estadística, que debe de recoger para cada alta el correspondiente CMBD, bien en soporte informático o escrito, debiendo el Gerente de cada Institución garantizar la conservación, custodia, disponibilidad y confidencialidad de la información contenida en las hojas Clínico-Estadísticas con arreglo a la legislación vigente (artículo 43 de la Constitución Española).

La Comunidad Valenciana fue la siguiente Comunidad Autónoma que definió un contenido y una normativa del CMBD (Orden 8-12-1992, DOGV 28-101992). En el País Vasco, mediante el Decreto 303/1992 de 3 de Noviembre (BOPV 1-12-1992), desarrollado por las resoluciones de 15 de Junio de 1993 (BOPV de 12 de Julio) y 30 de Julio de 1993 (BOPV de 24 de Agosto), se impone la recogida del CMBD. En la Comunidad Gallega, la Consejería de Sanidad regula el CMBD en la orden de 7 de Abril de 1993. En la Comunidad de Madrid, la Consejería de Salud del Servicio Regional de la Salud mediante el Proyecto SISTEMA, inicia en Julio de 1993 la implantación del CMBD. En Andalucía, es el Servicio Andaluz de Salud quien establece, mediante Resolución de 1993, la obligatoriedad del CMBD a partir del 1 de Enero de 1994. Por último, el Servicio Navarro de Salud, mediante Instrucción de 27 de mayo de 1994, establece la obligatoriedad del CMBD.

\subsection{Implantación del CMBD}

El objetivo principal para el conjunto del Sistema Nacional de Salud, al implantar el CMBD, era la obtención de bases de datos normalizados capaces de proporcionar una información asistencial válida, fiable, comparable y oportuna de todos los hospitales integrantes del Sistema Nacional de Salud, como elemento clave del Sistema de Información Asistencial de dichos hospitales y del conjunto del Sistema. (Rivero, 1992).

La estrategia de implantación del CMBD se basó en los siguientes prerequisitos:

a) existencia de una historia clínica única por paciente y hospital. 
b) garantizar que el CMBD fuera obtenible para todos los pacientes dados de alta en el hospital.

c) garantizar una adecuada extracción, codificación y verificación de los datos.

d) garantizar los recursos informáticos necesarios para la explotación del CMBD.

e) asegurar un adecuado análisis de la información generada por la explotación del CMBD.

\subsection{Características funcionales del CMBD}

El CMBD constituye una base de datos relativos y recogidos al alta de cada episodio de hospitalización, con las características de ser extensiva (recoge todas las altas hospitalarias) y homogénea en cuanto al tipo y codificación de las variables incluidas. Se puede decir que es el prototipo de base de datos administrativa, y que constituye el núcleo mínimo de información sobre cada enfermo que debe existir en todo sistema sanitario, siendo de utilidad tanto en el ámbito asistencial como de gestión. La ampliación de este conjunto mínimo de datos con algunas otras variables de interés, como pueden ser fecha de intervención quirúrgica, servicio de ingreso, servicio de alta, etc., o cualquier otra necesaria para cubrir unos objetivos específicos, se viene realizando a nivel interno en los propios hospitales o dentro de una Comunidad Autónoma, si bien la estructura de la base de datos que se envía trimestralmente al Sistema Nacional de Salud debe de ser la misma para todo el territorio español.

Podemos clasificar los datos que se recogen en datos administrativos y datos asistenciales (Tabla 2):

- Los datos administrativos, dentro de un mismo hospital y para el mismo individuo, permanecen constantes e identifican tanto al hospital como al paciente; podemos decir que son fijos para el mismo individuo tantas veces como sea ingresado en el mismo hospital.

- Los datos asistenciales o clínicos, son por el contrario, son propios y característicos de cada episodio, y por consiguiente, cambiantes cada vez que ingresa y es dado de alta en un hospital.

Este tipo de datos, según el INSALUD, debe de proporcionar una información válida y fiable, y es necesaria para la gestión eficiente de las instituciones sanitarias y para garantizar la calidad y eficacia de todos sus servicios. Permitirá, asimismo, conocer tanto los servicios prestados como los procesos atendidos en el hospital. El CMBD, por su disponibilidad y bajo coste relativo, es el principal soporte de información para medir los resultados de la atención hospitalaria, tanto de efectividad o de calidad (mortalidad, reingresos, complicaciones, etc.), 


\begin{tabular}{|l|l|}
\hline Datos adMinistrativos & Datos ASISTENCIALES \\
\hline Identificación del hospital & Fecha de ingreso \\
\hline Identificación del paciente & Circunstancias del ingreso \\
\hline Residencia & Diagnósticos \\
\hline Financiación & Procedimientos quirúrgicos y obstétricos \\
\hline Sexo & Otros procedimientos \\
\hline Fecha de nacimiento & Fecha de alta \\
\hline & Circunstancias del alta \\
\hline & Identificación médico responsable del alta \\
\hline
\end{tabular}

Tabla 2. Tipo de datos del CMBD

como de eficiencia (coste por proceso, duración de la estancia, etc.), permitiendo realizar comparaciones al respecto.

\subsection{Utilidades del CMBD}

La utilidad del CMBD como Sistema de Información asistencial radica en sus dos vertientes: Sistma de Recuperación de la Información y Sistema de Información propiamente dicho.

\subsubsection{Sistema de Recuperación de Información}

Es posible definirlo así, entendiendo como tal sistema, la base metodológica y tecnológica que permite proporcionar los documentos al usuario que los solicita. Su principal función es la de dar información sobre los documentos, que en este caso son las historias clínicas. La historia clínica constituye el documento primario, y para incluirse dentro del CMBD debe de sufrir un análisis documental. Mediante la indización de algunas características, se representa el contenido de los episodios asistenciales de la historia clínica; estas características propias de cada episodio asistencial son los ítems del CMBD, y constituyen el perfil del documento.

La recuperación del documento se hace formulando un perfil de búsqueda o condiciones exigidas en cualquiera de las variables o campos que forman parte del CMBD. Si el perfil de búsqueda coincide con el perfil del documento, se considera que el documento es pertinente, pudiendo acceder a la historia clínica a través de su número, que es único por paciente y forma parte del CMBD. 
Como tal sistema de recuperación de información, el CMBD se usa frecuentemente para poder averiguar los números de las historias de pacientes con unos determinados diagnósticos, o que se les ha practicado alguna intervención, pudiendo además exigir que estas condiciones se den en varones o mujeres, o en un grupo de edad determinado, o que hayan fallecido, etc.

De esta manera, servirá de base para poder realizar estudios más profundos (epidemiológicos, de control de calidad, etc.) que requieran recopilar datos que no están incluidos en la base de datos pero sí se encuentran en la historia clínica, o bien para seleccionar una muestra con las historias clínicas que necesiten ser revisadas.

\subsubsection{Sistema de Información}

El CMBD puede actuar también como Sistema de Información propiamente dicho, entendiendo como tal el proceso estadístico de los datos y la salida de unos resultados en forma de indicadores que permitan medir los resultados y los productos hospitalarios, así como conocer la actividad realizada por los Servicios y la morbilidad atendida en cada uno de ellos, incluso la detección de problemas potenciales de calidad e ineficiencia. En este sentido, es el sistema de información más ampliamente implantado y utilizado en los hospitales, no sólo en España sino en Europa y en Estados Unidos, por lo que constituye un sistema de información homogéneo y comparable entre hospitales y países. Destaca su gran potencia, en el sentido de que a partir de unos pocos datos o variables primarias, es capaz de crear otras variables secundarias y de proporcionar una gran cantidad de información y/o indicadores.

Las principales utilidades de este sistema de información son muchas, pero podemos destacar:

1. La primera se deriva del hecho de registrar las enfermedades y los procedimientos quirúrgicos relativos al paciente, mediante la Clasificación Internacional de Enfermedades (CIE-9-MC). Esto permite conocer la morbilidad hospitalaria, de gran interés para la planificación sanitaria y estudios epidemiológicos. Pero, más importante en la actualidad, es que se puede aplicar algún Sistema de Clasificación de Pacientes o de case mix que contenga los algoritmos de clasificación en forma de programa informático (GRD, PMC), y conocer el case mix (casuística) y qué produce el hospital y/o los servicios.

2. Conocimiento de la actividad asistencial desarrollada por los servicios, pero no sólo en lo que se refiere a cantidad sino también en lo referente a la complejidad de la demanda y resultados obtenidos.

3. Obtención de indicadores de gestión y de calidad asistencial. 
4. Conocimiento de la mortalidad hospitalaria.

5. Conocimiento de la estructura de la población atendida según sexo y edad.

6. Docencia e investigación, tanto clínica como epidemiológica. Los estudios descriptivos son sobe todo válidos cuando se efectúan sobre una base poblacional (provincia, comunidad autónoma, país), y los estudios etiológicos tratan de verificar hipótesis de asociación entre factores de riesgo y enfermedades. En este último caso, la información hospitalaria puede producir una distorsión (efecto Berkson) cuando la probabilidad de ingreso está condicionada por el factor de riesgo, pudiendo dar una falsa asociación positiva o negativa.

Además, todos los indicadores y la información resultante pueden ajustarse por el case mix o casuística, con lo que se elimina de esta manera la influencia de ésta como factor de confusión al comparar servicios u hospitales.

El establecimiento del CMBD en los hospitales ha supuesto el primer paso en el desarrollo de un sistema de información integrado (con información de contabilidad) en un intento de conseguir nuevas fórmulas para controlar los costes, los presupuestos clínicos o la planificación sanitaria a partir de una medida del producto hospitalario (sistemas de clasificación por case mix) mejor que las clásicas estancias. Hay que tener en cuenta que la información clínica adquiere una gran trascendencia en la gestión de un hospital, tanto en la relación con la propia gestión como en la relación con los denominados factores críticos de éxito de la empresa hospitalaria, y uno de los principales factores de éxito es la necesidad de contar con la existencia de Sistemas de Información que permitan seguir la estructura y producción del hospital, tanto en cantidad como en calidad. Si una definición de gestión es la información en acción, en la gestión de los servicios sanitarios la información clínico-asistencial es la búsqueda de las decisiones a tomar (Esteban, 1990).

La información sanitaria constituye uno de los pilares fundamentales para la gestión de servicios sanitarios, así como para la realización de estudios clínicos y epidemiológicos. No hay gestión eficiente sin planificación, y ésta no puede desarrollarse sin un sistema de información que permita la toma de decisiones. El hospital moderno es una institución de estructura compleja cuyo objetivo final es mejorar la salud de la población a la que presta servicio; para ello es condición imprescindible conocer las características de la misma, así como los motivos por los que acude. Antiguamente era el Libro de Registro de Ingresos y Altas, y actualmente el CMBD, la fuente de información útil para la obtención de dichos datos, tanto por la información que recoge y su obligatoriedad como por su accesibilidad y sencillez de manejo. 


\section{Conclusiones}

A grandes rasgos, podemos decir que en un hospital existen dos tipos de sistemas de información: sistema de información de tipo asistencial, que está integrado por el Conjunto Mínimo Básico de Datos (CMBD), y que con el uso de un agrupador de pacientes (GRD, PMC) permite clasificar a éstos y conocer la producción y el case mix hospitalario; la gran potencia de este sistema le viene determinada por la gran cantidad de información y de indicadores que se pueden obtener a partir de un número pequeño de variables o ítems: morbilidad atendida en el hospital, indicadores de gestión y de actividad (estancia media, estancia preoperatoria, $\mathrm{n}^{\circ}$ de altas), indicadores de resultados (mortalidad, reingresos, infecciones), etc., pudiendo obtener toda esta información con el objetivo de conocer el rendimiento a nivel global del hospital, o bien a nivel de cada servicio clínico.

En el otro lado, se pueden situar los sistemas de información de contabilidad analítica, donde se registran y asignan los costes, y que en este momento se puede decir que están implantados en la mayoría o todos los hospitales de la red del INSALUD (Proyecto SIGNO).

La tendencia actual es al cambio del modelo de gestión y presupuestación de los hospitales, en base a lo que se conoce como coste por proceso, y que implica un cambio profundo en el funcionamiento del hospital, sobre todo porque la gestión se centra ya en el producto final y no en los productos intermedios (estancias, UBAS, UPAS). Pero para llegar a esta situación, se necesita que los dos sistemas de información mencionados, junto con otro sistema que recoja todas las prestaciones y recursos asistenciales consumidos por un paciente durante su estancia hospitalaria, se integren en un único sistema de gestión hospitalaria. Sólo de esta manera es posible integrar costes y actividad y llegar a conocer el verdadero coste por proceso, y por tanto, cambiar el sistema actual de gestión.

El desarrollo e impulso de estos sistemas de información de coste por proceso vienen marcados por un hecho clave en los países desarrollados: el incremento del gasto sanitario. En España, por ejemplo, el gasto sanitario es el segundo capítulo en importancia del Presupuesto. En 1975, el gasto sanitario representaba el 3,9\% del P.I.B., y en 1996, casi el 6\%. Esta situación no es muy distinta de la del resto de los países de la Unión Europea, por lo que nos enfrentamos a una importante crisis de financiación sanitaria, ya que la demanda es imparable y los recursos son limitados. Se hace necesario, pues, un objetivo como es la contención del gasto sanitario, y la consecución de este objetivo lleva aparejadas una serie de medidas entre las que se encuentra la aplicación de otras técnicas de gestión sanitaria más eficaces como es el coste por proceso; pero para ello, debe de desarrollarse un sistema de información integrado por los tres subsistemas mencionados anteriormente, y en este sentido, tal sistema de información debe de eri-

Scire. 3 : 2 (jul.-dic. 1997) 115-130 
girse en la herramienta que proporcione una información válida y fiable para tomar decisiones correctas en el terreno de la gestión, financiación y planificación.

En España, existen ya en los hospitales tanto el CMBD como el sistema de contabilidad analítica, disponiendo también del sistema de medición de case mix GRD en todos los hospitales del INSALUD. El único eslabón que falta, es el desarrollo del sistema de registro de todos los servicios que recibe el paciente. En nuestro país se han llevado a cabo experiencias y proyectos para conocer el coste por proceso, entre las que podríamos destacar el trabajo de Mercé Casas realizado en hospitales catalanes con los GRD y el trabajo de Paloma Alonso en el Hospital de Móstoles con los PMC. Aparte de estas experiencias aisladas y algunas más, no existe en los hospitales españoles un sistema de información integrado que permita conocer el coste por proceso de forma normalizada.

Por otra parte, destacaríamos el hecho de que en España, en el ámbito sanitario, y en concreto en el hospitalario, la implantación de los sistemas de información ha llevado un considerable retraso con respecto a los demás países desarrollados y existe un vacío importante hasta 1990-1992, años en que los hospitales públicos comienzan a tener el CMBD de forma automatizada; posteriormente, en 1995, el INSALUD adquiere las licencias del agrupador de GRD a la empresa $3 \mathrm{M}$, y se quiere pasar ya al coste por proceso. Es decir, vemos que en pocos años y prácticamente de repente, se quiere tener funcionando un sistema de información hospitalario integrado; y esto entraña unos riesgos como son: falta de datos o datos escasamente válidos, falta de personal adecuado tanto para la recogida de datos como para el proceso y análisis de los datos, escasez de recursos informáticos, falta de mentalidad y sensibilización de los médicos para participar activamente en los flujos de información, y otros factores que afectan al proceso de obtención de una información fiable, válida y real.

Además de todo esto, hay que tener presente que en el coste por proceso se quiere implicar de forma activa a los médicos, sobre todo desde el punto de vista de la toma de decisiones en la gestión de sus servicios, y ello requerirá que éstos dispongan de una información totalmente fiable, ya que de lo contrario el fracaso de estos sistemas de información y de los nuevos modelos de gestión está asegurado. Solamente con un soporte de información estrictamente real y válida, los Servicios podrán mejorar su productividad y eficiencia, y tendrán capacidad real de gestionar tanto el proceso y sus componentes como los productos intermedios.

\section{Bibliografía}

Association of American Medical Colleges (1986). Evaluation of medical information science in medical education. // Journal of Medical Education. 61 (1986) 487-543.

Collen, M.F. (1988). HIS concepts, goals and objectives. // Towards new hospital infor-

Scire. 3 : 2 (jul.-dic. 1997) 115-130 
mation systems. The Netherlands : Elsevier Science Publishers, 1988, p. 3-9.

Esteban García, J.; Vicens Gómez, J. (1990). Información clínica y gestión hospitalaria. // Todo Hospital. 66 (1990) 25-28.

Gejo, J.M. ; Serra, M. ; Santiña, M. (1990). La información clínico asistencial: definición y aspectos a considerar. // Todo Hospital. 66 (1990) 13-16.

Gerneth, F. ; Haux, R. ; Selbman, H.K. (1991). On research subsystems and their integration in the computer supported part of hospital information systems. // Med. Inf. Lond. $16: 1$ (1991) 77-95.

Kaplan B. (1988). Development and acceptance of medical information systems: an historical overview. // J. Health Hum. Resour. Adm. 11 : 1 (1988) 9-29.

Korpman, R.A. (1991). Health care information systems. Patient centered integration is the key. // Clinics in Laboratory Medicine. 11 : 1 (1991) 203-220.

Regidor, E. (1991). Sistema de información sanitaria en la planificación.// Rev. San. Hig. Pub. 65 (1991) 9-16.

Rivero, A. (1992). Sistema de información basado en el CMBD.// Papeles Médicos. 1 (1992) 13-14.

Salvador Oliván, J.A. (1997). El sistema de información CMBD como herramienta de gestión y de control de calidad hospitalaria. Tesis Doctoral, Univ. de Zaragoza, 1997.

Selbmann, H.K. ; Pietsch-Breitfeld, B. (1990). Hospital information systems and quality assurance. // Quality Assurance in Health Care. 2 : 3-4 (1990) 335-344.

Terrada, M.L.; Peris, R. (1988). Lecciones de Documentación Médica. Uniersitat de Valencia, 1988.

World Health Organization (1990). The role of research and information systems in decision making for the development of human resources for health. // WHO Technical Report Series. 802 (1990)

Zwetsloot-Schonk, J.H.M. ; Snitker, P. ; Vandenbroucke, J.P. ; Bakker, A.R. (1989). Using hospital information systems for clinical epidemiological research. // Med. Inform. 14 : 1 (1989) 53-62 